\title{
BMJ Open Cross-sectional comparison of health- related quality of life and other features in people with and without objective and subjective binge eating using a general population sample
}

\author{
Natalie Li, ${ }^{1}$ Deborah Mitchison, ${ }^{2}$ Stephen Touyz, ${ }^{3}$ Phillipa Hay ${ }^{\oplus}$
}

To cite: Li N, Mitchison D, Touyz S, et al. Cross-sectional comparison of health-related quality of life and other features in people with and without objective and subjective binge eating using a general population sample. BMJ Open 2019;9:e024227. doi:10.1136/ bmjopen-2018-024227

- Prepublication history for this paper is available online. To view these files, please visit the journal online (http://dx.doi org/10.1136/bmjopen-2018024227).

Received 16 May 2018 Revised 21 January 2019 Accepted 22 January 2019

Check for updates

(c) Author(s) (or their employer(s)) 2019. Re-use permitted under CC BY-NC. No commercial re-use. See rights and permissions. Published by BMJ.

${ }^{1}$ School of Medicine, Western Sydney University, Penrith, New South Wales, Australia ${ }^{2}$ Psychology Department, Macquarie University, Sydney, New South Wales, Australia

${ }^{3}$ Clinical Psychology, The University of Sydney, Sydney, New South Wales, Australia

${ }^{4}$ Translational Health Research Institure, School of Medicine, Western Sydney, Penrith, New South Wales, Australia

Correspondence to Phillipa Hay;

P.Hay@westernsydney.edu.au

\section{ABSTRACT}

Objectives Evidence suggests that while objective binge eating (OBE) and subjective binge eating (SBE) differ in the amount of food consumed, both are associated with impairment in people with eating disorders. However, only $\mathrm{OBE}$ is accounted for in the diagnostic criteria of eating disorders. This study compared the sociodemographic profile and burden of OBE versus SBE at a population level. Design Population-based survey.

Participants A representative sample of 3028 men and women. Participants were categorised into four groups based on their reporting of binge eating in the past 3 months: non-binge eating group (no OBE or SBE), OBE group, SBE group and OSBE group (both OBE and SBE). Outcome measures Demographics (age, genderand body mass index, BMI), binge eating, distress, weight/ shape overvaluation and health-related quality of life. Groups were compared on sociodemographic information, overvaluation and health-related quality of life. The OBE and SBE groups were also compared on the distress related to binge eating.

Results No differences were found between the SBE group and $\mathrm{OBE}$ group in age, gender, $\mathrm{BMI}$, mental healthrelated quality of life and overvaluation (all $p>0.05$ ). However, differences were found in the OSBE participants, namely that they were younger, had a higher mean BMI, lower mental health-related quality of life and higher overvaluation of weight/shape than the non-binge-eating participants (all $p<0.001$ ). Proportions of participants who reported distress related to binge eating in the OBE and SBE groups also did not differ $(p=0.678)$.

Conclusion There is little difference in the demographic profile or burden of people who engage in OBE versus SBE, supporting the proposed inclusion of SBE in the diagnostic criteria for eating disorders in International Classification of Diseases-11. People who experience both OBE and SBE may experience a relatively higher eating disorder severity and impairment.

\section{INTRODUCTION}

Objective binge eating (OBEs) episodes are a core diagnostic criteria for both bulimia nervosa $(\mathrm{BN})$ and binge eating disorder
Strengths and limitations of this study

- The study comprised a representative sample of the general population of adults, attenuating the gender bias towards young women that is frequently associated in eating disorder investigations.

- Study questionnaires were administered at interview by trained personnel, decreasing vulnerability to reporting bias.

- The cross-sectional nature of the study precludes longitudinal patient follow-up.

- All study participants were selected from the predominantly metropolitan state of South Australia, potentially reducing nationwide or international applicability.

(BED), and also often occur in patients with anorexia nervosa (AN). ${ }^{1}$ An OBE is currently defined in the fifth edition of the Diagnostic and Statistical Manual of Mental Disorders (DSM-5) as being characterised by the sensation of a loss of control (LOC) over eating in a discrete time frame, during which the amount of food consumed is 'definitely larger than what most people would eat' under similar circumstances. ${ }^{1}$ However, not only is the definition of a 'definitely large' amount of food subject to the practitioner's clinical judgement, ${ }^{2}$ this current criteria exclude the phenomenon of subjective binge eating (SBE). This is a form of binge eating closely related to an OBE which is also associated with LOC, and which many people with eating disorders and in the general population experience. In contrast to OBEs, SBEs involve the consumption of a small or moderate amount of food that is perceived by the individual as a binge. ${ }^{2}$

Studies comparing OBE and SBE have demonstrated considerable similarities between clinical features, outcomes and 
impairment of people with these two closely related behaviours; ${ }^{2-4}$ however, there is still international dispute regarding SBEs' formal inclusion into the DSM criteria for eating disorders.

Results from recent studies have demonstrated no differences between SBE and OBE in regard to levels of associated depression, substance abuse, general psychological distress and interpersonal problems, ${ }^{34}$ and both have shown clinically significant psychological impacts of binge eating. ${ }^{5}$ Furthermore, studies conducted by Palavras et al examining the epidemiology of SBEs and OBEs have also found similar sociodemographic profiles, for example in regard to marital status and age ${ }^{56}$ Several limitations are observed in these studies however, including a lack of samples that are representative of the general population, and frequent exclusion of men and of older individuals. ${ }^{56}$ Nonetheless, research to date suggests that when the criterion of size is removed from the definition of a binge episode, the common attribute of LOC between OBEs and SBEs becomes the remaining defining parameter. ${ }^{2}$ The importance of LOC has also been suggested to be the key determinant of psychological problems, with a diminished relative importance of amount of food consumed. ${ }^{7}$ Ultimately, this has led researchers to question the clinical significance of size distinction in binge episodes. ${ }^{3}$

Furthermore, a 2008 study of undergraduate American women has found SBEs to be a more common experience than OBEs $(16.7 \%$ and $6.4 \%$, respectively $),{ }^{8}$ and could have potential clinical importance in regard to treatment of related eating disorders. ${ }^{9}$ A recent cohort study of 218 individuals with BN or BED by Castellini et al demonstrated that a higher frequency of SBEs predicted lower rates of recovery with cognitive behavioural therapy, suggesting that careful consideration of SBE as a treatment target may be important for achieving treatment goals. ${ }^{9}$ Previous studies have also showed that not only may SBEs be implicated in the treatment of other EDs, individuals with SBEs are less responsive to treatment than those with OBEs, ${ }^{47}$ and are more likely to experience persisting negative affect and psychopathology. ${ }^{10}$ As binge eating is a key symptom in the DSM diagnostic guidelines for $\mathrm{BN}, \mathrm{BED}$ and $\mathrm{AN}$ binge eating/purge subtype $(\mathrm{AN} \mathrm{B} / \mathrm{P}),{ }^{1}$ the inclusion of SBEs into diagnostic criteria is therefore particularly relevant for treatment and service access.

The extent which eating disorders are associated with overvaluation is also important to consider particularly given that it is a central feature across eating disorders. ${ }^{11}$ Overvaluation is the excessive importance of weight or shape on one's self-evaluation, ${ }^{11}$ and is a core DSM-5 diagnostic criterion for $\mathrm{AN}$ and $\mathrm{BN}$, but not for BED. ${ }^{12}$ However, prior research has shown that patients with BED have reported similar levels of overvaluation as patients with $\mathrm{BN},{ }^{13}$ and subsequent studies have argued that overvaluation should be included as a BED diagnostic specifier. ${ }^{14}$ There is still some controversy regarding its inclusion however, as another study comparing OBEs and SBEs demonstrated that neither were associated with significant difference in shape or weight concern. ${ }^{15}$ This discrepancy highlights a need for further research into this topic, particularly given that much of the existing data on overvaluation in BED has been limited by their sample bias towards women. ${ }^{11} 1617$

Currently, the WHO has recognised the potential clinical significance of SBEs, and has removed the essential requirement for the size of a binge to be large in the International Classification Diseases (ICD) for BEDs. ${ }^{18}$ In the proposed ICD-11 criteria, a binge eating episode is a "distinct period of time during which the individual experiences a subjective loss of control over eating, eating notably more or differently than usual, and feels unable to stop eating or limit the type or amount of food eaten'. Multiple studies as described above support this revision to the WHO ICD-11 scheme. ${ }^{56}$ However, we cannot be confident of generalisation to the general population as these studies have often been conducted with clinical and community samples with restricted demographic representation. It is particularly important to include both males and older people in studies of binge eating, as disorders involving this behaviour have been demonstrated to exhibit less of a gender bias towards young females than other eating disorders. ${ }^{12} 19$

The current study uses a representative community adult sample, and aims to determine whether there are differences in sociodemographic profiles (age, sex and educational attainment), levels of distress regarding binge eating episodes, overvaluation and health-related quality of life (HRQoL) between people with recurrent OBEs only, people with solely recurrent SBEs and people with combined OBEs and SBEs. Given previous research findings, we expect that based on current literature, there will be no differences in health outcomes between participants with OBE only and those with SBE only. However, we did hypothesise that those who experience combined OBEs and SBEs will have poorer health outcomes than people with solely OBEs or solely SBEs.

\section{METHODS}

\section{Sampling procedures}

This study analysed data collected in 2016 from the Health Omnibus Survey, which is a face-to-face interview survey conducted annually by Harrison Health Research. The Health Omnibus Survey has collated South Australian health data for government and non-government organisations since 1991, with individual organisations paying to include health questions that are relevant to their research. ${ }^{20}$

Household samples included in the Health Omnibus Survey were randomly selected from metropolitan and rural areas in proportion to their population size as based on the Australian Bureau of Statistics collector districts. Ten households within each collector district were then selected, and the resident older than 15 years who most recently had their birthday was chosen to partake in the 
interview. If the selected participant was not at home, a maximum of six repeat visits was made to the chosen household. The sample was non-replacement; meaning that if the selected individual could not be interviewed, no other persons from the household were interviewed in their place. Construct validity and interview feasibility was ensured through pilot testing of 50 members of the general public. Due to the pragmatic constraints of large household surveys, verbal consent was obtained from all interview participants and additional written parental consent from participants aged $<18$ years. In 2016, 5300 individuals were in the initial household sample, 80 houses were vacant, 1129 refused, 736 could not be contacted, remainder were ineligible. Total interviews were 3047 , thus the overall response rate was $58.4 \%$ and participation rate $68 \%$ (initial eligible sample excluding those with non-contact after six attempts). The most cited reason for non-participation was refusal (32\%).

\section{Measures}

\section{Demographic information and body mass index}

Four parameters were analysed as indicators of participant demographics. These included gender, age, highest level of educational attainment and participant body mass index (BMI; $\left.\mathrm{kg} / \mathrm{m}^{2}\right)$ as calculated from their self-reported height and weight. Educational attainment was assessed through participant self-nomination from the following categories: still in school, left school before age 15, left school after age 15, left school after age 15 but still studying, trade qualification or apprenticeship, certificate or diploma (less than or equal to 1 year completed), certificate or diploma (more than 1 year completed), or bachelor degree or higher.

\section{Objective and subjective binge eating}

Endorsement of objective binge eating episodes (OBE) within the last 3 months was assessed by the following interview question: 'I would now like to ask you about episodes of overeating. By overeating, or binge eating, I mean eating an unusually large amount of food in one go and at the time feeling that your eating was out of control. Over the past three months how often have you overeaten (in this way)? Would you say...1. Not at all, 2. Less than weekly, 3. Once a week, or 4. Two or more times a week'. Similarly, the interviewer established the endorsement of SBE episodes with the following question: 'Over the past 3 months have you felt your eating was out of control when others might not agree the amount of food was unusually large (eg, 2-3 pieces of bread)? Would you say...1. Not at all, 2. Less than weekly, 3. Once a week, 4. Two or more times a week'. For both OBE and SBE episodes, the interviewer was instructed to observe that the respondent reported that they could not prevent themselves from overeating, or could not stop eating once they had started. For the purposes of this study, recurrent OBE and SBEs were defined as occurring weekly or more over the past 3 months. The OSBE group had both OBE and SBE occurring weekly for 3 months.

\section{Distress related to binge eating}

Participants who reported either recurrent OBEs or SBEs were asked two follow-up questions to establish the level of distress they experienced in relation to both their recurrent OBE and separately their recurrent SBE. Participants indicated either 'not at all', 'yes, a little' or 'yes, a lot'. In this study, marked distress was recorded when the respondent endorsed 'yes a lot'.

Health-related quality of life

Participants' HRQoL was established using the abridged 12-item Medical Outcomes Study Short Form (SF-12) questionnaire. This questionnaire measures physical function and mental health through the assessment of various domains including participants' occupational, social and emotional health. ${ }^{21}$ The questionnaire involves two subscales, a mental health component score (MCS) and physical health component score (PCS), which were then transformed into T-scores with a mean of 50 and SD of 10 . The SF-12 has been shown to be a valid and reliable tool in assessing HRQoL in the Australian population, ${ }^{22}$ with higher scores indicating better HRQoL. ${ }^{21}$

\section{Overvaluation}

Overvaluation of weight and/or shape is the extent to which weight and shape influences one's self-evaluation, and was measured in this study using a question modelled from the Eating Disorder Examination ${ }^{23}$ : 'On a scale of $0-6$, where 0 is Not at all important and 6 is Extremely or the most important issue, how important an issue has your weight and/or your shape been to how you think about (judge or view) yourself as a person in the past threemonths?' This item has been used previously in population studies to examine weight/shape concerns. ${ }^{24}$

\section{Data transformation and analysis}

Data were inspected for normality and completeness. The rate of missing data was $<0.5 \%$ for all variables with the exception of BMI where it was $8 \%$. This was low and no imputation or adjustment was made. Data were weighted based on an individual's probability of selection, and then reweighted to the population distribution derived from the 2015 Estimated Resident Population, from the Australian Bureau of Statistics. The comparative frequency of $\mathrm{OBE}, \mathrm{SBE}$ and both $\mathrm{OBE}$ and $\mathrm{SBE}$ in the past 3 months was tested using the $\mathrm{X}^{2}$ test. Prevalence data are reported as percentages with $95 \%$ CI.calculated using the NewcombeWilson method without continuity correction using an Excel syntax. ${ }^{25}$

For the purposes of further data analysis, participants were grouped based on their endorsement of OBEs and SBEs: 1. Participants who reported neither OBE or SBE in the past 3 months (non-binge eating group), 2. Participants who reported only OBE in the past 3 months (OBE group), 3. Participants who reported only SBE in the past 3 months (SBE group) and 4. Participants who reported both OBE and SBE in the past 3 months (OSBE group). In regard to educational attainment, the collated 
data were merged to form three major categories: 1 . No tertiary qualifications, 2 . Trade or certificate qualification and 3. Bachelor degree or higher. For the purposes of data analysis, these categories were then merged to form three major groups: no tertiary qualifications, trade or certificate qualification, or bachelor degree or higher. $\mathrm{X}^{2}$ tests were used to compare distress related to binge eating between the OBE group and the SBE group, and to compare gender and educational attainment distribution between all four groups. Distress related to binge eating was only compared between OBE and SBE groups. We did not assess distress related to binge eating in the OSBE group as they were asked distress related to SBE and distress related to OBE, and thus included four groups of people with distress related to OBE, distress related to SBE, distress related to both OBE and SBE and no distress with either. Kruksal-Wallis tests were used to compare levels of overvaluation between the four binge eating groups, and Mann-Whitney U Test for posthoc analyses.

Two univariate analysis of variances (ANOVAs) with Tukey posthoc tests $(\mathrm{p}<0.05)$ were performed to analyse differences in age and BMI between the four groups. HRQoL was compared between the four groups using two multivariate ANOVAs (MANCOVA), with mental health (MCS) and physical health composite scale (PCS) scores as dependent variables, and BMI, age, gender and education as covariates. Differences between groups were considered significant at $\mathrm{p}<0.05$. The Bonferroni correction was used for posthoc tests. All data analyses were performed using SPSS V.21.

\section{Patient and public involvement}

This was a general population study and patients were not involved in the design of the study.

\section{RESULTS}

\section{Prevalence of OBE, SBE and OSBE}

Overall, the sample consisted of $\mathrm{n}=3028$, including $\mathrm{n}=2578$ (85.1\%; $95 \%$ CI $83.8 \%$ to $86.4 \%)$ who met criteria for the non-binge eating group (reported neither OBE or SBE), $\mathrm{n}=353(11.7 \%$; $95 \%$ CI $10.6 \%$ to $12.9 \%)$ who met criteria for the OBE group (reported OBE only), $\mathrm{n}=20$ $(0.7 \% ; 95 \%$ CI $0.4 \%$ to $1.0 \%)$ who met criteria for the SBE group (reported SBE only) and $\mathrm{n}=77(2.5 \% ; 95 \%$ CI $2.0 \%$ to $3.3 \%$ ) who met criteria for the OSBE group (reported both OBE and SBE).

\section{Demographic features and binge eating}

As shown in table 1, a significant main effect of group on age was observed, $\mathrm{F}(3,3023)=27.11, \mathrm{p}<0.001$. Posthoc tests revealed that participants in the OBE $(p<0.001)$ and OSBE $(p<0.001)$ groups were on average significantly younger than participants in the non-binge eating group (medium effect sizes). There were also significant differences in BMI observed between groups, F (3, $2746)=16.01, \mathrm{p}<0.001$, with posthoc tests demonstrating higher mean BMI in the OSBE group when compared with all other groups (medium to large effect sizes) and the OBE group also demonstrated a significantly higher mean BMI when compared with the non-binge eating group (small effect size). No differences in gender distribution or educational attainment were observed between groups (all $\mathrm{p}>0.05$ ).

\section{Overvaluation}

As shown in table 1, a main effect of group on overvaluation was observed. Posthoc testing demonstrated significantly higher levels of overvaluation in the OBE group (median=4.0) and OSBE groups (median=4.0) when compared with the non-binge eating group (median=3.0), $\mathrm{U}=359274.00, \quad \mathrm{Z}=-6.051, \quad \mathrm{p}<0.001, \quad \mathrm{r}=-0.11$ and $\mathrm{U}=71848.50, \quad \mathrm{Z}=-3.89, \quad \mathrm{p}<0.001, \quad \mathrm{r}=0.07$, respectively. The OSBE group also scored higher on overvaluation compared with the SBE group (median=2.9), $\mathrm{U}=495.50$, $\mathrm{Z}=-2.14, \mathrm{p}=0.03, \mathrm{r}=0.22$. Effect sizes were small. No significant differences were revealed between the OBE and SBE groups, $\mathrm{p}=0.06$.

\section{Distress related to binge eating}

As shown in table 1, no significant differences in distress related to binge eating were demonstrated between OBE and SBE groups (all $\mathrm{p}>0.05$ ).

\section{Health-related quality of life}

A main effect of group on MCS scores was observed, F (3, $2714)=7.8, \mathrm{p}<0.001$. On posthoc testing, the OSBE group demonstrated significantly lower MCS scores on average when compared with the OBE (small effect size) and non-binge eating (medium effect size) groups. No effect of group was observed for PCS scores (see table 2).

\section{DISCUSSION}

The present study investigated similarities and differences in four groups of people recruited from a community sample, namely people who did not binge eat (non-binge eating group), people who reported regular OBEs (OBE group), people who reported regular SBEs (SBE group) and people who reported both OBEs and SBEs (OSBE group). We found significant similarities between the OBE and SBE groups across all measures. This supports previous research indicating that subjective binge eating is associated with similar health-related consequences and eating-related psychopathology as DSM-5 recognised OBEs. ${ }^{3-7} 18$

With regard to demographic profiles, participants of the SBE and OBE groups were of a similar age, which is consistent with prior research generally indicating a marked demographic similarity between SBE and OBE groups. ${ }^{45}{ }^{26}$ Study results also showed that those in the non-binge eating group were on average 7 years and 10 years older than participants of the OBE and OSBE groups, respectively. This is in contrast to the results of Dalle Grave et al which found non-binge eating participants 
Table 1 Descriptive statistics between groups for gender, age, body weight and educational attainment

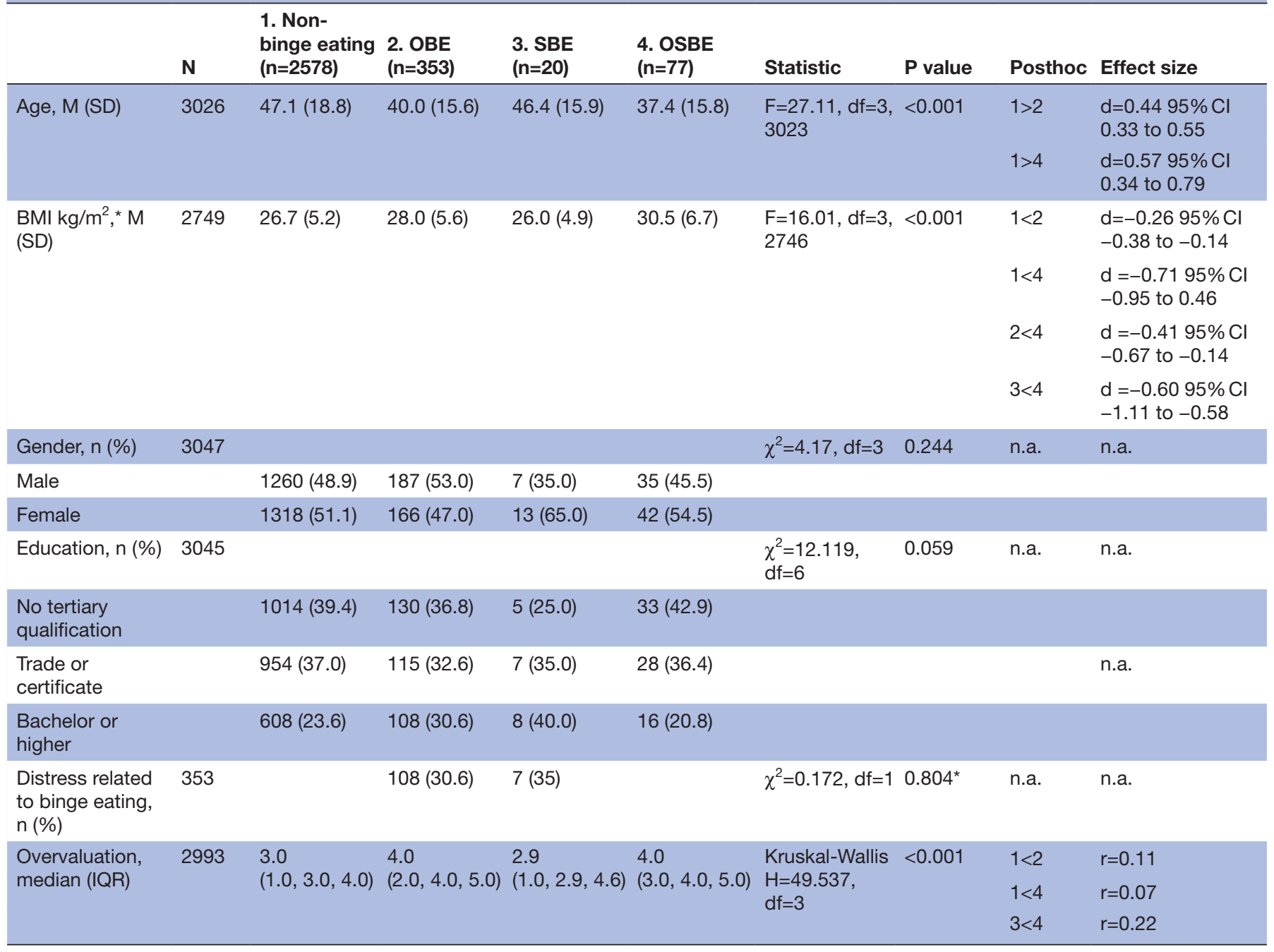

*Distress was only compared between OBE only (353) and SBE only (20) groups, Fisher exact test used.

BMI, body mass index; d, Cohen 's d; OBE, recurrent objective binge eating alone; n.a., not applicable; OSBE, mixed objective and subjective recurrent binge eating episodes; $r=Z$ /sqare root $(n 1+n 2)$; SBE, recurrent subjective binge eating alone.

were of similar age to those in the OBE and SBE groups. ${ }^{27}$ A possible explanation for this difference is that the Dalle Grave study had a very small sample size (33-36 participants) in each binge-eating group and was less representative than the sample in the present study. It also suggests that binge eating may disproportionately affect younger people, although other evidence suggests that the age of onset for BED is older than for other eating disorders (eg, $\mathrm{AN}$ and $\mathrm{BN})$. We also found no effect of gender. Current literature is divided on this subject, with some research demonstrating a small female gender bias, ${ }^{28}$ and other studies that report an equal prevalence of binge eating among men and women. ${ }^{29}$ However, the overall trend of research to date does suggest that gender bias towards

Table 2 Effect of binge group on health-related quality of life

\begin{tabular}{|c|c|c|c|c|c|c|c|}
\hline & $\begin{array}{l}\text { 1. Non-binge eating } \\
(n=2318)\end{array}$ & $\begin{array}{l}\text { 2. OBE } \\
(n=319)\end{array}$ & $\begin{array}{l}\text { 3. SBE } \\
(n=18)\end{array}$ & $\begin{array}{l}\text { 4. OSBE } \\
(n=67)\end{array}$ & MANCOVA & Posthoc & Cohen's d, 95\% Cl \\
\hline MCS & $53.0(8.3)$ & $51.6(8.1)$ & $51.1(7.4)$ & $47.4(11.6)$ & $F(3,2714)=7.8, p<0.001$ & $\begin{array}{l}1<4 \\
2<4\end{array}$ & $\begin{array}{l}0.67,0.42 \text { to } 0.91 \\
0.38,-0.14 \text { to } 0.90\end{array}$ \\
\hline PCS & $49.3(9.8)$ & $49.4(9.7)$ & $50.3(5.9)$ & $48.5(9.7)$ & $F(3,2714)=1.2, p=0.293$ & & n.a. \\
\hline
\end{tabular}

MANCOVA analyses include BMI, age, gender and education as covariates.

ANOVA, analysis of variance; MANCOVA, multivariate ANOVA; MCS, mental health component score; OBE, recurrent objective binge eating alone; n.a., not applicable; OSBE, mixed objective and subjective recurrent binge eating episodes; PCS, physical health component score; $\mathrm{SBE}$, recurrent subjective binge eating alone. 
young women is less prevalent in BED than for other eating disorders. ${ }^{19}{ }^{20}$ Likewise, there were no differences observed in our study based on educational attainment, similar to previous research with BED patients ${ }^{426}$ and where differences were found effect sizes were not large.

Regarding BMI, no significant differences were demonstrated between participants of the OBE and SBE groups, which might be surprising given that these behaviours differ based on the amount of food consumed. While a 2010 paper by Mond and colleagues indicated that regular endorsement of OBEs was associated with a significantly higher BMI than regular endorsement of SBEs, ${ }^{30}$ the results of our study are generally consistent with prior data. ${ }^{4529}$ On the other hand, as expected, participants who experienced both OBEs and SBEs were found to have a significantly higher average BMI than participants who only reported OBEs or SBEs, and participants who only reported OBEs had a significantly higher average BMI than participants who did not binge eat. However, effect sizes were not large.

Study results showed that participants of the OBE and SBE groups demonstrated remarkable similarity with regard to distress related to binge eating. This is consistent with current literature, which has found the size of the binge to be irrelevant to the extent of general psychological distress experienced. ${ }^{36}$ The reason for this finding has been postulated to be due to LOC over eating, which is a shared phenomenon between OBEs and SBEs that is thought to be more important than binge size in determining the degree of impairment experienced. ${ }^{67}$

Similarly, there was also no difference between the OBE and SBE groups with respect to HRQoL, which is in concordance with prior research. ${ }^{29}$ While we found poorer mental health among individuals with OSBE when compared with individuals of the $\mathrm{OBE}$ and non-binge eating groups, supporting our preliminary hypothesis that the OSBE group would likely have poorer health outcomes than other groups, the effect sizes were not large. Notably, a previous study of 214 women found that relative to non-binge eating patients, $\mathrm{SBE}$ and $\mathrm{OBE}$ participants showed markedly poorer health outcomes in the mental health domain ${ }^{31}$; however, our study did not specifically demonstrate poorer quality of life among participants who only endorsed recurrent (weekly) OBEs or SBEs when compared with the non-binge eating group. Previous research from our group in the South Australian population has found the impact of weekly OBEs on mental HRQoL appears to be reducing since 1998, but when OBEs occurred two times weekly there was significant impact on mental HRQoL. ${ }^{32}$

With regard to overvaluation, this study showed results to be comparable between the OBE and SBE groups, which is in support of current literature describing a similar level of weight and shape concern between individuals who regularly engage in OBEs versus SBEs. ${ }^{40}$ Furthermore, and as would be expected based on prior findings, body shape overvaluation was higher among participants in our study who endorsed any type of binge eating than in non-binge eating participants. While previous studies show that endorsement of both OBEs and SBEs does not appear to increase shape and weight concern, ${ }^{30}$ our study did demonstrate significantly higher levels of overvaluation in the OSBE group when compared with the SBE and non-binge eating groups, which again supports our preliminary hypothesis.

While there is currently little research specifically examining the effect of engaging in both OBEs and SBEs, our results also showed that people who endorsed both types of binge eating (the OSBE group) generally demonstrated the poorest outcomes in health-related measures such as BMI, HRQoL and overvaluation. It is possible that participants who reported both OBEs and SBEs engage in more frequent binge eating, although as binge eating was assessed as a categorical variable, this cannot be ascertained. This is a group that warrants further investigation.

Finally, it is of note that endorsing only OBEs was far more prevalent $(11.7 \%)$ than endorsing both OBEs and SBEs $(2.5 \%)$ or SBEs alone $(0.7 \%)$. These findings are in contrast to prior research demonstrating that SBEs were more commonly experienced than OBEs, ${ }^{80}$ with SBE versus OBE rates reported to be as high as $16.7 \%$ and $6.4 \%$, respectively. ${ }^{8}$ Demographic and temporal differences in samples may explain this finding as well as the possibility that the size of binge eating episodes may be increasing with time. However, this speculation requires further research.

\section{Strengths and limitations}

One of the main strengths of this study is its large sample size and inclusion of a representative sample of the general population of adults, which is in contrast to many previous studies that have had a greater bias towards young women from treatment settings in their sample selection. ${ }^{56}$ This is particularly relevant in BEDs where prevalence in men has been shown to be approximately equal to those in women. ${ }^{1920} 29$ Another study strength is its use of trained personnel to conduct the survey interviews, increasing the validity and reliability of study results. Furthermore, the specific analysis of OSBE endorsement is a novel aspect of this paper, as investigating a binge eating subpopulation is rarely exclusively studied despite its poorer health outcomes. However, several limitations of this study should also be noted. First, despite the large sample size, there were a relatively low number of participants who reported SBEs, which may have decreased the power to find significant differences between groups, and there were uneven group sizes. Second, the cross-sectional nature of the study means that there is no longitudinal follow-up, therefore precluding any causal inferences regarding the association between binge eating and the outcomes measured. Third, as mentioned previously, binge eating was not assessed as a continuous variable, which precluded our ability to compare and assess the effects of frequency of binge episodes on health outcomes. Fourth, OBE and SBE participants were characterised by recurrence in the last 3 months; however, the 
SF-12 measures quality of life for the past 4 weeks; thus, there was only partial assessment of quality of life over the designated time period. Fifth, BMI was calculated from self-reported height and weight, and this lack of standardised measuring may have resulted in data inaccuracies. Finally, all study participants were from the largely metropolitan state of South Australia, so results may not generalise nationwide or internationally.

\section{Study implications}

Overall, our finding that SBE and OBE participants are comparable across all measured indices is consistent with prior research demonstrating that the size of the binge eating episode is a relatively less important factor in determining mental and physical health implications. ${ }^{367}$ This is supportive of the new ICD-11 revision proposal, which moves to discard the parameter of binge size in the classification of binge eating in an effort to improve rates of detection and treatment for all binge eating subpopulations.

Another implication of the current findings is that further investigation is required for the binge eating subpopulation that endorses both SBEs and OBEs. Results demonstrating that OSBE participants had the worst health outcomes indicate a need for greater awareness of this subpopulation among health researchers and clinicians, as this should lead to improvements in treatment.

Additionally, the finding that sex differences are negligible in the endorsement of BED has significant implications in challenging the historical view that eating disorders are endorsed by a predominantly young, female population. Currently, the vast majority of eating disorder prevention programmes are targeted towards young adolescent and adult women $;{ }^{33}$ however, the current findings support the need for intervention programmes that are more relevant and targeted to men. There is thus a greater role for improvement of health literacy in the community and health professionals to increase awareness of BED in men and improve rates of male treatment seeking and referral.

\section{Future research}

In regard to future research, the results of this study open up several potential avenues for further exploration. In particular, there needs to be further studies in representative community samples using a longitudinal design to examine the clinical impacts of SBEs compared with OBEs on HRQoL and other outcomes. As well, further research into the characteristics and health implications of individuals with both OBEs and SBEs should be considered.

\section{CONCLUSION}

The current finding that there is a strong similarity in clinical features between individuals experiencing OBEs and individuals experiencing SBEs has a significant implication in the diagnosis and treatment of SBEs. Thus, in concordance with prior research, this paper supports the inclusion of SBEs in the diagnostic criteria for eating disorders characterised by recurrent binge eating, as currently proposed by the ICD-11 Feeding and Eating Disorders Working Group. The findings also highlight the need for a more gender-equal approach to eating disorder prevention, as well as a more thorough and specific investigation into the health implications of OSBE endorsement.

Contributors NL contributed to the design and led the aims of the study, participated in data analysis and was prime author of the manuscript; DM supervised the design, ran the multivariate analyses and reviewed the manuscript; ST contributed to the design and reviewed the manuscript; PH led the design, contributed to the aims of the study, participated in data analysis and reviewed the manuscript.

Funding This work was supported by internal Western Sydney University research funds (provided to Professor Hay) and internal University of Sydney research funds (provided to Professor Touyz).

Competing interests $\mathrm{PH}$ receives/has received sessional fees and lecture fees from the Australian Medical Council, Therapeutic Guidelines publication and New South Wales Institute of Psychiatry and royalties/honoraria from Hogrefe and Huber McGraw Hill Education and Blackwell Scientific Publications, Biomed Central and PlosMedicine and she has received research grants from the NHMRC and ARC. She is Deputy Chair of the National Eating Disorders Collaboration Steering Committee in Australia (2012-) and Member of the ICD-11 Working Group for Eating Disorders (2012-2018) and was Chair Clinical Practice Guidelines Project Working Group (Eating Disorders) of RANZCP (2012-2015). She has prepared a report under contract for Shire Pharmaceuticals in regard to Binge Eating Disorder (BED; July 2017) and received honoraria and travel support for teaching Psychiatrists from Shire Pharmaceuticals (2018). All views in this paper are her own. ST is the Chair of the Shire Australasian BED Advisory Committee. He has received both research grants and travel grants from Shire and has received consultancy fees for both commissioned reports and speaking engagements. He is also in receipt of royalties from McGraw Hill for contribution to text books.

Patient consent for publication Not required.

Ethics approval Ethical approval for the survey including verbal consent was granted by the University of Adelaide Human Research Ethics Committee number H-097-2010.

Provenance and peer review Not commissioned; externally peer reviewed.

Data sharing statement Unpublished data are available from Professor Hay for collaborative research projects.

Open access This is an open access article distributed in accordance with the Creative Commons Attribution Non Commercial (CC BY-NC 4.0) license, which permits others to distribute, remix, adapt, build upon this work non-commercially, and license their derivative works on different terms, provided the original work is properly cited, appropriate credit is given, any changes made indicated, and the use is non-commercial. See: http://creativecommons.org/licenses/by-nc/4.0/.

\section{REFERENCES}

1. American Psychiatric Association. Diagnostic and statistical manual of mental disorders: DSM-5. 5th edn. Arlington: American Psychiatric Publishing Inc, 2013.

2. Wolfe BE, Baker CW, Smith AT, et al. Validity and utility of the current definition of binge eating. Int J Eat Disord 2009;42:674-86.

3. Niego SH, Pratt EM, Agras WS. Subjective or objective binge: is the distinction valid? Int J Eat Disord 1997;22:291-8.

4. Brownstone LM, Bardone-Cone AM, Fitzsimmons-Craft EE, et al. Subjective and objective binge eating in relation to eating disorder symptomatology, negative affect, and personality dimensions. Int $J$ Eat Disord 2013;46:66-76.

5. Palavras MA, Morgan CM, Borges FM, et al. An investigation of objective and subjective types of binge eating episodes in a clinical sample of people with co-morbid obesity. J Eat Disord 2013;1:26.

6. Palavras MA, Hay PJ, Lujic S, et al. Comparing symptomatic and functional outcomes over 5 years in two nonclinical cohorts characterized by binge eating with and without objectively large episodes. Int J Eat Disord 2015;48:1158-65. 
7. Latner JD, Clyne C. The diagnostic validity of the criteria for binge eating disorder. Int J Eat Disord 2008;41:1-14.

8. Luce KH, Crowther JH, Pole M. Eating Disorder Examination Questionnaire (EDE-Q): norms for undergraduate women. Int J Eat Disord 2008;41:273-6.

9. Castellini G, Mannucci E, Lo Sauro C, et al. Different moderators of cognitive-behavioral therapy on subjective and objective binge eating in bulimia nervosa and binge eating disorder: a three-year follow-up study. Psychother Psychosom 2012;81:11-20.

10. Goldschmidt AB, Accurso EC, Crosby RD, et al. Association between objective and subjective binge eating and psychopathology during a psychological treatment trial for bulimic symptoms. Appetite 2016;107:471-7.

11. Hrabosky JI, Masheb RM, White MA, et al. Overvaluation of shape and weight in binge eating disorder. J Consult Clin Psychol 2007;75:175-80.

12. Hay P, Girosi F, Mond J. Prevalence and sociodemographic correlates of DSM- 5 eating disorders in the Australian population. $J$ Eat Disord 2015;3:19.

13. Masheb RM, Grilo CM. Binge eating disorder: a need for additional diagnostic criteria. Compr Psychiatry 2000;41:159-62.

14. Mitchison D, Rieger E, Harrison C, et al. Indicators of clinical significance among women in the community with binge-eating disorder symptoms: Delineating the roles of binge frequency, body mass index, and overvaluation. Int J Eat Disord 2018;51:165-9.

15. Fitzsimmons-Craft EE, Ciao AC, Accurso EC, et al. Subjective and objective binge eating in relation to eating disorder symptomatology, depressive symptoms, and self-esteem among treatmentseeking adolescents with bulimia nervosa. Eur Eat Disord Rev 2014;22:230-6.

16. Grilo CM, Hrabosky JI, White MA, et al. Overvaluation of shape and weight in binge eating disorder and overweight controls: refinement of a diagnostic construct. J Abnorm Psychol 2008;117:414-9.

17. Harrison C, Mond J, Rieger E, et al. Correlates of binge eating with and without overvaluation of weight or shape: further evidence from a general population sample of women. Adv Eat Disord 2015;3:20-33.

18. World Health Organisation. The 11th Revision of the International Classification of Diseases (ICD-11). 2018 http://www.who.int/ classifications/icd/revision/en/ (Accessed 27 Aug 2018).

19. Mitchison D, Mond J. Epidemiology of eating disorders, eating disordered behaviour, and body image disturbance in males: a narrative review. J Eat Disord 2015;3:20.
20. Health GoSADo. Health omnibus survey 2008. 2008 http://www. health.sa.gov.au/pros/Default.aspx?tabid (Accessed 18 Mar 2011).

21. Ware J, Kosinski M, Keller SD. A 12-Item Short-Form Health Survey: construction of scales and preliminary tests of reliability and validity. Med Care 1996;34:220-33.

22. Lim LL, Fisher JD. Use of the 12-item short-form (SF-12) Health Survey in an Australian heart and stroke population. Qual Life Res 1999;8(1-2):1-8.

23. Cooper Z, Fairburn C. The eating disorder examination: a semi-structured interview for the assessment of the specific psychopathology of eating disorders. Int J Eat Disord 1987;6:1-8.

24. Hay P, Mitchison D, Collado AEL, et al. Burden and health-related quality of life of eating disorders, including Avoidant/Restrictive Food Intake Disorder (ARFID), in the Australian population. J Eat Disord 2017;5:21.

25. Newcombe RG. Interval estimation for the difference between independent proportions: comparison of eleven methods. Stat Med 1998:17:873-90.

26. Mulders-Jones B, Mitchison D, Girosi F, et al. Socioeconomic correlates of eating disorder symptoms in an australian populationbased sample. PLoS One 2017;12:e0170603.

27. Dalle Grave R, Calugi S, Marchesini G. Objective and subjective binge eating in underweight eating disorders: associated features and treatment outcome. Int J Eat Disord 2012;45:370-6.

28. Striegel-Moore RH, Rosselli F, Perrin N, et al. Gender difference in the prevalence of eating disorder symptoms. Int $J$ Eat Disord 2009;42:471-4

29. Hudson JI, Hiripi E, Pope HG, et al. The prevalence and correlates of eating disorders in the National Comorbidity Survey Replication. Biol Psychiatry 2007;61:348-58.

30. Mond JM, Latner JD, Hay PH, et al. Objective and subjective bulimic episodes in the classification of bulimic-type eating disorders: another nail in the coffin of a problematic distinction. Behav Res Ther 2010;48:661-9.

31. Vallance J. The relationship between eating disorder psychopathology and quality of life within a non-clinical sample. Christchurch: University of Canterbury, 2006.

32. Mitchison D, Touyz S, González-Chica DA, et al. How abnormal is binge eating? 18-Year time trends in population prevalence and burden. Acta Psychiatr Scand 2017;136:147-55.

33. Stice E, Becker CB, Yokum S. Eating disorder prevention: current evidence-base and future directions. Int J Eat Disord 2013;46:478-85. 